DFTT 06/2000

INFNCA-TH0002

hep-ph/0002120

\title{
Spin effects in the fragmentation of a transversely polarized quark
}

\author{
M. Anselmino ${ }^{1}$ and F. Murgia ${ }^{2}$ \\ ${ }^{1}$ Dipartimento di Fisica Teorica, Università di Torino and \\ INFN, Sezione di Torino, Via P. Giuria 1, I-10125 Torino, Italy \\ ${ }^{2}$ Istituto Nazionale di Fisica Nucleare, Sezione di Cagliari \\ and Dipartimento di Fisica, Università di Cagliari \\ C.P. 170, I-09042 Monserrato (CA), Italy
}

\begin{abstract}
:
An azimuthal dependence of pions produced in polarized Deep Inelastic Scattering, $\gamma^{*} p^{\uparrow} \rightarrow \pi X$, has been recently observed and might be related to the so-called Collins effect. We discuss in details, for a general spin configuration of the nucleon, the kinematics of the process and methods of extracting information on the fragmentation properties of a polarized quark. Assuming that the observed azimuthal dependence is indeed due to Collins effect, we derive a lower bound estimate for the size of the quark analysing power, which turns out to be large.
\end{abstract}




\section{Introduction}

Semi-inclusive polarized Deep Inelastic Scattering combines in a unique way spin properties of quarks inside hadrons: a leading hadron inside a current jet should result from a quark inside the polarized nucleon which - after the well known QED interaction with the lepton - fragments into the hadron. The number of, say, pions produced in such a process depends both on the quark polarized distribution and fragmentation functions. If one considers transverse polarizations, new, yet unexplored properties become accessible, which have been or might soon be observed in several experiments in progress.

An example of this is the azimuthal dependence - with respect to the $\gamma^{*}$ direction - of pions produced in $\ell p \rightarrow \ell^{\prime} \pi X$ processes, with unpolarized leptons and polarized protons, recently observed by HERMES [1] and by SMC [2] collaborations; if confirmed, this asymmetry would prove a novel feature of the fragmentation process of a transversely polarized quark, namely its dependence on the direction of the intrinsic transverse momentum of the hadron inside the jet. Such spin and $\boldsymbol{k}_{\perp}$ dependence was predicted some time ago [3], but only recently became object of a careful investigation. It might be the crucial key towards a complete understanding of other single spin asymmetries observed in $p p^{\uparrow} \rightarrow \pi X$ processes [4], and related to quark transverse motion [5]-[7].

We concentrate here on such a single spin effect in DIS, giving explicit expressions for the measurable observables, considering different initial spin states of the nucleon, discussing the implications of the existing data and extracting from them some phenomenological information. We do not attempt to explain the data using particular models for the unknown distribution or fragmentation functions, as other authors do [8]-11]. Our work is somewhat related to that of Ref. [12], with the difference that we focus our attention on direct cross-section measurements, rather than weighted integrals, we discuss in details the experimental measurements to be performed and describe how to obtain qualitative information from the data. We use the QCD parton model language, at leading twist, allowing for transverse quark motion in the fragmentation process only (Collins effect [3]); we do not discuss contributions from higher twist distribution or correlation functions.

\section{Collins fragmentation function}

Let us consider a quark with momentum $\boldsymbol{p}_{q}$ and a transverse polarization vector $\boldsymbol{P}_{q}\left(\boldsymbol{p}_{q} \cdot \boldsymbol{P}_{q}=0\right)$ which fragments into a hadron with momentum $\boldsymbol{p}_{h}=z \boldsymbol{p}_{q}+\boldsymbol{p}_{T}$ $\left(\boldsymbol{p}_{q} \cdot \boldsymbol{p}_{T}=0\right)$ : according to Collins suggestion [3] the fragmentation function for the $q \rightarrow h+X$ process can be written as

$$
D_{h / q}\left(\boldsymbol{p}_{q}, \boldsymbol{P}_{q} ; z, \boldsymbol{p}_{T}\right)=D_{h / q}\left(z, p_{T}\right)+\frac{1}{2} \Delta^{N} D_{h / q}\left(z, p_{T}\right) \frac{\boldsymbol{P}_{q} \cdot\left(\boldsymbol{p}_{q} \times \boldsymbol{p}_{T}\right)}{\left|\boldsymbol{p}_{q} \times \boldsymbol{p}_{T}\right|}
$$

where $D_{h / q}\left(z, p_{T}\right)$ is the unpolarized fragmentation function. Notice that - as required by parity invariance - the only component of the polarization vector which 
contributes to the spin dependent part of $D$ is that perpendicular to the $q-h$ plane; in general one has, see Fig. 1:

$$
\boldsymbol{P}_{q} \cdot \frac{\boldsymbol{p}_{q} \times \boldsymbol{p}_{T}}{\left|\boldsymbol{p}_{q} \times \boldsymbol{p}_{T}\right|}=P_{q} \sin \left(\varphi_{P_{q}}-\varphi_{h}\right) \equiv P_{q} \sin \Phi_{C}
$$

where $P_{q}=\left|\boldsymbol{P}_{q}\right|$ and we have defined the Collins angle $\Phi_{C} \equiv \varphi_{P_{q}}-\varphi_{h}$. It is obvious that any longitudinal (with respect to $\boldsymbol{p}_{q}$ ) component of $\boldsymbol{P}_{q}$ does not contribute to Eq. (11).

When studying single spin asymmetries one considers differences of cross-sections with opposite transverse spins; by reversing the nucleon spin all polarization vectors, including those of quarks, change sign and the quantity which eventually contributes to single spin asymmetries is:

$$
D_{h / q}\left(\boldsymbol{p}_{q}, \boldsymbol{P}_{q} ; z, \boldsymbol{p}_{T}\right)-D_{h / q}\left(\boldsymbol{p}_{q},-\boldsymbol{P}_{q} ; z, \boldsymbol{p}_{T}\right)=\Delta^{N} D_{h / q}\left(z, p_{T}\right) \frac{\boldsymbol{P}_{q} \cdot\left(\boldsymbol{p}_{q} \times \boldsymbol{p}_{T}\right)}{\left|\boldsymbol{p}_{q} \times \boldsymbol{p}_{T}\right|}
$$

which implies the existence of a quark analysing power for the fragmentation process $q \rightarrow h+X:$

$$
\begin{aligned}
A_{q}^{h}\left(\boldsymbol{p}_{q}, \boldsymbol{P}_{q} ; z, \boldsymbol{p}_{T}\right) & =\frac{D_{h / q}\left(\boldsymbol{p}_{q}, \boldsymbol{P}_{q} ; z, \boldsymbol{p}_{T}\right)-D_{h / q}\left(\boldsymbol{p}_{q},-\boldsymbol{P}_{q} ; z, \boldsymbol{p}_{T}\right)}{D_{h / q}\left(\boldsymbol{p}_{q}, \boldsymbol{P}_{q} ; z, \boldsymbol{p}_{T}\right)+D_{h / q}\left(\boldsymbol{p}_{q},-\boldsymbol{P}_{q} ; z, \boldsymbol{p}_{T}\right)} \\
& =\frac{\Delta^{N} D_{h / q}\left(z, p_{T}\right)}{2 D_{h / q}\left(z, p_{T}\right)} \frac{\boldsymbol{P}_{q} \cdot\left(\boldsymbol{p}_{q} \times \boldsymbol{p}_{T}\right)}{\left|\boldsymbol{p}_{q} \times \boldsymbol{p}_{T}\right|} \equiv A_{q}^{h}\left(z, p_{T}\right) \frac{\boldsymbol{P}_{q} \cdot\left(\boldsymbol{p}_{q} \times \boldsymbol{p}_{T}\right)}{\left|\boldsymbol{p}_{q} \times \boldsymbol{p}_{T}\right|}
\end{aligned}
$$

A simple explicit expression of the function $\Delta^{N} D\left(z,\left\langle p_{T}\right\rangle\right)$ was obtained in Ref. [7] by fitting the data on single spin asymmetries in $p p^{\uparrow} \rightarrow \pi X$ processes [4] under the assumption that they entirely originate from Collins effect alone. It results that the quark analysing power $A_{q}^{\pi}\left(z, p_{T}\right)$ has to reach the limiting value -1 at large $z$ in order to describe the data.

However, such a result depends also on the chosen expressions for the polarized quark distribution functions, in particular on their large $x$ behaviour. The same study was repeated in Ref. [13], allowing for unusual large $x$ behaviours of the longitudinally polarized distribution functions $\Delta q(x)$ and imposing Soffer's inequality bound [14] on the transversely polarized distribution functions $\Delta_{T} q(x)$ or $h_{1}(x)$; the E704 data [4] could then be fitted leading to a constant, negative, $z$ independent, value of $A_{q}^{\pi}\left(z, p_{T}\right)$.

We discuss now how to extract information on $A_{q}^{h}\left(z, p_{T}\right)$ from semi-inclusive DIS scattering processes, and use the first available data [1, 2] to obtain a lower bound estimate of its magnitude.

\section{Single transverse spin asymmetries in $\gamma^{*} p^{\uparrow} \rightarrow \pi X$}

Let us consider the semi-inclusive DIS process $\ell p \rightarrow \ell^{\prime} \pi X$ in the $\gamma^{*}-p$ center of mass frame, with the kinematical configuration of Fig. 2, where we have chosen 
$x-z$ as the lepton scattering plane with the $\gamma^{*}$ moving along the positive $z$-axis and with:

$$
\hat{\boldsymbol{y}}=\frac{\ell \times \ell^{\prime}}{\left|\ell \times \ell^{\prime}\right|} \quad \hat{\boldsymbol{x}}=\hat{\boldsymbol{y}} \times \hat{\boldsymbol{z}}
$$

Let

$$
x=\frac{Q^{2}}{2 p \cdot q}, \quad y=\frac{Q^{2}}{s x}, \quad s=(\ell+p)^{2}, \quad z=\frac{p \cdot p_{h}}{p \cdot q},
$$

be the usual DIS variables.

In the configuration of Fig. 2 - neglecting intrinsic motion of quarks inside the initial proton - the elementary interaction, $\gamma^{*}+q, \boldsymbol{P}_{q} \rightarrow q^{\prime}, \boldsymbol{P}_{q^{\prime}}$, takes place along the $\hat{z}$-axis: $\boldsymbol{P}_{q}$ is the initial quark transverse spin-polarization vector and the final quark transverse spin-polarization $\boldsymbol{P}_{q^{\prime}}$ is fixed by the QED elementary dynamics and is given by:

$$
\begin{aligned}
\left(\boldsymbol{P}_{q^{\prime}}\right)_{x} & =\frac{-2(1-y)}{1+(1-y)^{2}}\left(\boldsymbol{P}_{q}\right)_{x} \\
\left(\boldsymbol{P}_{q^{\prime}}\right)_{y} & =\frac{2(1-y)}{1+(1-y)^{2}}\left(\boldsymbol{P}_{q}\right)_{y} .
\end{aligned}
$$

Notice that changing $\boldsymbol{P}_{q}$ into $-\boldsymbol{P}_{q}$ changes the sign of $\boldsymbol{P}_{q^{\prime}}$. The factor $\left|\boldsymbol{P}_{q^{\prime}}\right| /\left|\boldsymbol{P}_{q}\right|=$ $2(1-y) /\left[1+(1-y)^{2}\right] \equiv \hat{D}_{N N}$ is the so-called depolarization factor; apart from this magnitude reduction, $\boldsymbol{P}_{q}$ and $\boldsymbol{P}_{q^{\prime}}$ only differ for the sign of the $x$-component which corresponds to the situation described in Fig. 3.

Let us consider the general case of a nucleon with some transverse spin-polarization vector

$$
\boldsymbol{P}=P\left(\cos \varphi_{P}, \sin \varphi_{P}, 0\right) .
$$

If we denote by $P_{\uparrow}\left(P_{\downarrow}\right)$ the probability for the nucleon to have spin parallel (antiparallel) to $\boldsymbol{P}$ the degree of transverse polarization of the nucleon is given by:

$$
P=|\boldsymbol{P}|=P_{\uparrow}-P_{\downarrow} .
$$

The measured cross-sections for a proton with transverse spin-polarization vector $\boldsymbol{P}$ can be written as

$$
\begin{aligned}
\frac{d \sigma^{\ell+p, \boldsymbol{P} \rightarrow \ell^{\prime}+h+X}}{d x d y d z d \boldsymbol{p}_{T}} & =\sum_{q}\left[f_{\uparrow} \boldsymbol{P}_{(x)} \frac{d \hat{\sigma}_{q}^{\uparrow}}{d y} D_{h / q}\left(\boldsymbol{P}_{q^{\prime}} ; z, \boldsymbol{p}_{T}\right)\right. \\
& \left.+f_{\downarrow} \boldsymbol{P}_{(x)} \frac{d \hat{\sigma}_{q}^{\downarrow}}{d y} D_{h / q}\left(-\boldsymbol{P}_{q^{\prime}} ; z, \boldsymbol{p}_{T}\right)\right]
\end{aligned}
$$

where $f_{\uparrow(\downarrow)}^{\boldsymbol{P}}$ is the density number of transversely polarized quarks with polarization $\uparrow=\hat{\boldsymbol{P}}(\downarrow=-\hat{\boldsymbol{P}})$ inside a proton with polarization vector $\boldsymbol{P}$ (notice the difference between $\boldsymbol{P}$ and $\hat{\boldsymbol{P}}=\boldsymbol{P} / P$ :

$$
f_{\uparrow}^{\boldsymbol{P}}=P_{\uparrow} f_{\uparrow}^{\uparrow}+P_{\downarrow} f_{\uparrow}^{\downarrow} \quad f_{\downarrow}^{\boldsymbol{P}}=P_{\uparrow} f_{\downarrow}^{\uparrow}+P_{\downarrow} f_{\downarrow}^{\downarrow} .
$$


$D_{h / q}\left( \pm \boldsymbol{P}_{q^{\prime}} ; z, \boldsymbol{p}_{T}\right)$ is the fragmentation function of the final quark with a polarization vector $\pm \boldsymbol{P}_{q^{\prime}}$ resulting from the $\ell+q, \pm \hat{\boldsymbol{P}} \rightarrow \ell^{\prime}+q^{\prime}$ interaction:

$$
D_{h / q}\left(\boldsymbol{P}_{q^{\prime}} ; z, \boldsymbol{p}_{T}\right)=\frac{d \hat{\sigma}_{q}^{\uparrow \rightarrow \uparrow} D_{h / q^{\uparrow}}+d \hat{\sigma}_{q}^{\uparrow \rightarrow \downarrow} D_{h / q^{\downarrow}}}{d \hat{\sigma}_{q}^{\uparrow \rightarrow \uparrow}+d \hat{\sigma}_{q}^{\uparrow \rightarrow \downarrow}}
$$

and

$$
D_{h / q}\left(-\boldsymbol{P}_{q^{\prime}} ; z, \boldsymbol{p}_{T}\right)=\frac{d \hat{\sigma}_{q}^{\downarrow \rightarrow \uparrow} D_{h / q^{\uparrow}}+d \hat{\sigma}_{q}^{\downarrow \rightarrow \downarrow} D_{h / q^{\downarrow}}}{d \hat{\sigma}_{q}^{\downarrow \rightarrow \uparrow}+d \hat{\sigma}_{q}^{\downarrow \rightarrow \downarrow}},
$$

where $d \hat{\sigma}_{q}^{\uparrow \rightarrow \uparrow}$ stands for $d \hat{\sigma}^{\ell+q, \uparrow \rightarrow \ell^{\prime}+q, \uparrow} / d y$, and so on; $\boldsymbol{P}_{q^{\prime}}$ is explicitely given in Eqs. (7) and (8), taking $\boldsymbol{P}_{q}=\hat{\boldsymbol{P}}$. As there is no single spin asymmetry in the elementary interaction one has $d \hat{\sigma}_{q}^{\uparrow \rightarrow \uparrow}+d \hat{\sigma}_{q}^{\uparrow \rightarrow \downarrow}=d \hat{\sigma}_{q}^{\uparrow}=d \hat{\sigma}_{q}^{\downarrow \rightarrow \uparrow}+d \hat{\sigma}_{q}^{\downarrow \rightarrow \downarrow}=d \hat{\sigma}_{q}^{\downarrow}=d \hat{\sigma}_{q}^{u n p}$. Notice also that $d \hat{\sigma}_{q}$ depends on the quark flavour only via its factorized charge, $d \hat{\sigma}_{q}=e_{q}^{2} d \hat{\sigma}$.

Similarly

$$
\begin{aligned}
\frac{d \sigma^{\ell+p,-} \boldsymbol{P}_{\rightarrow \ell^{\prime}+h+X}}{d x d y d z d \boldsymbol{p}_{T}} & =\sum_{q}\left[f_{\uparrow}^{-} \boldsymbol{P}_{(x)} \frac{d \hat{\sigma}_{q}^{\uparrow}}{d y} D_{h / q}\left(\boldsymbol{P}_{q^{\prime}} ; z, \boldsymbol{p}_{T}\right)\right. \\
& \left.+f_{\downarrow}^{-} \boldsymbol{P}_{(x)} \frac{d \hat{\sigma}_{q}^{\downarrow}}{d y} D_{h / q}\left(-\boldsymbol{P}_{q^{\prime}} ; z, \boldsymbol{p}_{T}\right)\right]
\end{aligned}
$$

From Eqs. (3), (11) and (15) one has:

$$
\begin{aligned}
2 \frac{d \sigma^{\ell+p \rightarrow \ell^{\prime}+h+X}}{d x d y d z d \boldsymbol{p}_{T}} & =\frac{d \sigma^{\ell+p, \boldsymbol{P} \rightarrow \ell^{\prime}+h+X}}{d x d y d z d \boldsymbol{p}_{T}}+\frac{d \sigma^{\ell+p,-}}{d x d y d z d \boldsymbol{p}_{T}} \\
& =\sum_{q} f_{q / p}(x) \frac{d \hat{\sigma}_{q}}{d y} 2 D_{h / q}\left(z, p_{T}\right)
\end{aligned}
$$

and

$$
\begin{aligned}
& \frac{d \sigma^{\ell+p, \boldsymbol{P} \rightarrow \ell^{\prime}+h+X}}{d x d y d z d \boldsymbol{p}_{T}}-\frac{d \sigma^{\ell+p,-}-\boldsymbol{P} \rightarrow \ell^{\prime}+h+X}{d x d y d z d \boldsymbol{p}_{T}} \\
= & \sum_{q} h_{1 q}(x) \frac{d \hat{\sigma}_{q}}{d y} \Delta^{N} D_{h / q}\left(z, p_{T}\right) P \frac{\boldsymbol{P}_{q^{\prime}} \cdot\left(\boldsymbol{p}_{q^{\prime}} \times \boldsymbol{p}_{T}\right)}{\left|\boldsymbol{p}_{q^{\prime}} \times \boldsymbol{p}_{T}\right|} .
\end{aligned}
$$

where $h_{1 q}=f_{\uparrow}^{\uparrow}-f_{\uparrow}^{\downarrow}$ is the transverse spin distribution function. The single spin asymmetry for the process $\ell p^{\uparrow} \rightarrow \ell^{\prime} h X$ is then given by:

$$
\begin{aligned}
& A_{N}^{h}\left(x, y, z, \boldsymbol{P}_{q^{\prime}}, \boldsymbol{p}_{T}\right)=\frac{d \sigma^{\ell+p, \boldsymbol{P} \rightarrow \ell^{\prime}+h+X}-d \sigma^{\ell+p,-} \boldsymbol{P}_{\rightarrow \ell^{\prime}+h+X}}{d \sigma^{\ell+p, \boldsymbol{P} \rightarrow \ell^{\prime}+h+X}+d \sigma^{\ell+p,-} \boldsymbol{P}_{\rightarrow \ell^{\prime}+h+X}} \\
& =\frac{\sum_{q} e_{q}^{2} h_{1 q}(x) \Delta^{N} D_{h / q}\left(z, p_{T}\right)}{2 \sum_{q} e_{q}^{2} f_{q / p}(x) D_{h / q}\left(z, p_{T}\right)} P \frac{\boldsymbol{P}_{q^{\prime}} \cdot\left(\boldsymbol{p}_{q^{\prime}} \times \boldsymbol{p}_{T}\right)}{\left|\boldsymbol{p}_{q^{\prime}} \times \boldsymbol{p}_{T}\right|} \text {. }
\end{aligned}
$$


Using Eqs. (2) and (7), (\$) the above asymmetry can be written as

$$
A_{N}^{h}\left(x, y, z, \Phi_{C}, p_{T}\right)=\frac{\sum_{q} e_{q}^{2} h_{1 q}(x) \Delta^{N} D_{h / q}\left(z, p_{T}\right)}{2 \sum_{q} e_{q}^{2} f_{q / p}(x) D_{h / q}\left(z, p_{T}\right)} \frac{2(1-y)}{1+(1-y)^{2}} P \sin \Phi_{C} \text {. }
$$

The value of $\sin \Phi_{C}$ depends on the direction of $\boldsymbol{P}_{q^{\prime}}$ : from Eqs. (77) and (8) or their graphical representation in Fig. 3 one can express the Collins angle $\Phi_{C}$ in terms of measurable angles:

$$
\Phi_{C}=\pi-\varphi_{P}-\varphi_{h}
$$

where $\varphi_{P}$ and $\varphi_{h}$ are respectively the azimuthal angles of the proton polarization vector and of the produced hadron; we always refer to the choice of axes of Fig. 2 .

Some comments might be useful.

- Eq. (19) simplifies for pion production if one assumes that transverse sea quark polarization in a nucleon is negligible, $h_{1 \bar{q}} \simeq 0$, and if one neglects fragmentation of non-valence quarks into a pion; that is, if one assumes, using also isospin and charge conjugation invariance:

$$
\begin{aligned}
D_{\pi^{+} / u} & =D_{\pi^{-} / d}=D_{\pi^{+} / \bar{d}}=D_{\pi^{-} / \bar{u}} \equiv D_{\pi / q} \\
2 D_{\pi^{0} / u} & =2 D_{\pi^{0} / d}=2 D_{\pi^{0} / \bar{u}}=2 D_{\pi^{0} / \bar{d}} \equiv D_{\pi / q} \\
D_{\pi^{+} / d} & =D_{\pi^{-} / u}=D_{\pi^{+} / \bar{u}}=D_{\pi^{-} / \bar{d}} \simeq 0
\end{aligned}
$$

and similarly for the $\Delta^{N} D$. Eq. (23) is justified at the large $z$ values for which data are available; moreover, for $\pi^{+}$, the contribution of $d$ and $\bar{u}$ quarks are further suppressed by the corresponding smaller distribution functions.

In this case Eq. (19) reads $(i=+,-, 0)$ :

$$
A_{N}^{\pi^{i}}\left(x, y, z, \Phi_{C}, p_{T}\right)=\frac{h_{i}(x)}{f_{i}(x)} A_{q}^{\pi}\left(z, p_{T}\right) \frac{2(1-y)}{1+(1-y)^{2}} P \sin \Phi_{C}
$$

where:

$$
\begin{gathered}
i=+: \quad h_{+}=4 h_{1 u} \quad f_{+}=4 f_{u / p}+f_{\bar{d} / p} \\
i=-: \quad h_{-}=h_{1 d} \quad f_{-}=f_{d / p}+4 f_{\bar{u} / p} \\
i=0: \quad h_{0}=4 h_{1 u}+h_{1 d} \quad f_{0}=4 f_{u / p}+f_{d / p}+4 f_{\bar{u} / p}+f_{\bar{d} / p} .
\end{gathered}
$$

- The asymmetries (24) are factorized into the product of five different physical quantities, each of which is smaller in magnitude than 1 ; it is remarkable that the existing data [1, 2], hint at values of $A_{N}$ of the order of a few percents. Out of the five factors, three - the transverse polarization $P$, the depolarization factor and $\sin \Phi_{C}-$ are known or measurable separately; the other two $-h_{i} / f_{i}$ and the quark analysing power $A_{q}^{\pi}=\Delta^{N} D_{\pi / q} / 2 D_{\pi / q}$ - are not known. The measured values of $A_{N}$ yield information on the product of these two quantities. However - and we shall attempt this in the sequel - some upper bounds for $h_{1}$ are known [14], and we should be able to obtain lower bound estimates for the size of the quark analysing power. 
- In order to cumulate data collected at different kinematical values, one should integrate the cross-sections (11) and (15), for example over the relevant $x$ and $y$ regions; in this case one obtains:

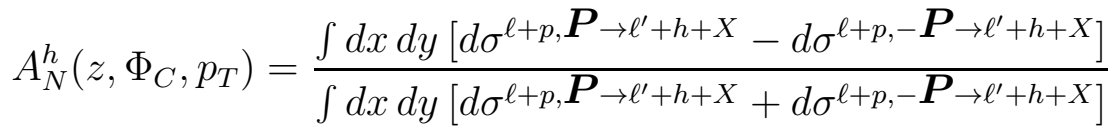

$$
\begin{aligned}
& =\frac{\sum_{q} \int d x d y e_{q}^{2} h_{1 q}(x) 2(1-y) /\left(x y^{2}\right) \Delta^{N} D_{h / q}\left(z, p_{T}\right) P \sin \Phi_{C}}{2 \sum_{q} \int d x d y e_{q}^{2} f_{q / p}(x)\left(1+(1-y)^{2}\right) /\left(x y^{2}\right) D_{h / q}\left(z, p_{T}\right)} .
\end{aligned}
$$

Notice that $P$ depends on $x$ and $y$, as we shall discuss in the next Section.

The analogue of Eq. (24) can be written as:

$$
A_{N}^{\pi^{i}}\left(z, \Phi_{C}, p_{T}\right)=\frac{I_{N_{i}}}{I_{D_{i}}} A_{q}^{\pi}\left(z, p_{T}\right) \sin \Phi_{C}
$$

with

$$
I_{N_{i}}=\int d x d y h_{i}(x) \frac{2(1-y)}{x y^{2}} P
$$

and

$$
I_{D_{i}}=\sum_{q} \int d x d y f_{i}(x) \frac{1+(1-y)^{2}}{x y^{2}},
$$

where $h_{i}$ and $f_{i}$ are given in Eqs. (25)-(27). In factorizing the quark analysing power $A_{q}^{\pi}$ in Eq. (29) we have neglected the smooth $y$ dependence of the fragmentation functions induced by $\mathrm{QCD} Q^{2}$-evolution.

\section{Measurements of $\boldsymbol{A}_{N}$ in particular cases}

Eq. (19) holds at leading twist - contributions decreasing as inverse powers of $Q$ have been neglected - and leading order in the parton transverse motion, in the sense that we have taken into account quark transverse momenta only in places where neglecting them would give zero value for $A_{N}$ : the quarks inside the initial fast proton are all collinear and the observed $\boldsymbol{p}_{T}$ of the pion arises entirely as transverse momentum in the fragmentation process.

In Eqs. (19) and (28) $\boldsymbol{P}$ is the proton polarization transverse to its direction in the $\gamma^{*}-p$ center of mass frame $(\boldsymbol{P} \cdot \boldsymbol{q}=0)$ : it is the only component of the polarization which contributes to $A_{N}$. In realistic situations the proton spin-polarization vector is not perpendicular to $\boldsymbol{q}$; there are two typical configurations in performed or planned experiments:

a) The proton spin-polarization vector, $\boldsymbol{P}_{L}$, is longitudinal with respect to the lepton direction in the laboratory frame, where the proton is at rest (Fig. 4).

b) The proton spin-polarization vector, $\boldsymbol{P}_{T}$, is transverse with respect to the lepton direction in the laboratory frame, where the proton is at rest (Fig. 5). 
Let us consider these two cases separately.

a) - Longitudinal proton polarization, $\boldsymbol{P}_{L}$

In this case the magnitude of the active component of the proton spin-polarization vector - i.e. the component which contributes to $A_{N}$ - is given by (see Fig. 4):

$$
P=P_{L} \sin \theta_{\gamma},
$$

with $\left(m_{N}\right.$ is the proton mass)

$$
\sin \theta_{\gamma}=2 m_{N}\left[\frac{\left(s-m_{N}^{2}\right) x y(1-y)-m_{N}^{2} x^{2} y^{2}}{\left(s-m_{N}^{2}\right)^{2} y^{2}+4 m_{N}^{2}\left(s-m_{N}^{2}\right) x y}\right]^{1 / 2} \simeq \frac{2 m_{N} x}{Q} \sqrt{1-y} .
$$

In the last expression we have neglected terms of $O\left(m_{N}^{3} / Q^{3}\right)$.

Eq. (33) shows how a longitudinal nucleon polarization can contribute to single transverse spin asymmetries in semi-inclusive DIS processes only at moderate values of $Q$ and in events for which the factor $2 x \sqrt{1-y}$ is not too small; this is the case of HERMES measurements [1]. It also indicates that higher twist contributions might be as important as leading twist ones, as discussed in Refs. [9, 11.

Fig. 4 shows how the azimuthal angle of $\boldsymbol{P}_{L}$ is 0 , so that, from Eq. (20),

$$
\Phi_{C}=\pi-\varphi_{h} .
$$

The factor $P \sin \Phi_{C}$ for a longitudinal proton polarization, assuming $P_{L}=1$, is then

$$
P \sin \Phi_{C} \simeq \frac{2 m_{N} x}{Q} \sqrt{1-y} \sin \varphi_{h} .
$$

b) - Transverse proton polarization, $\boldsymbol{P}_{T}$

In this case it is more elaborate to obtain the value of $P$, the magnitude of the polarization vector component orthogonal to $\boldsymbol{q}$, starting from a polarization vector $\boldsymbol{P}_{T}$ orthogonal to the lepton momentum, in the laboratory frame (see Fig. 5); one can show that

$$
P=P_{T}\left[\cos ^{2} \theta_{\gamma} \cos ^{2} \phi_{P}^{\ell^{\prime}}+\sin ^{2} \phi_{P}^{\ell^{\prime}}\right]^{1 / 2}
$$

where $\phi_{P}^{\ell^{\prime}}$ is the difference between the azimuthal angle of $\boldsymbol{P}_{T}$ and $\boldsymbol{\ell}^{\prime}$, measured around the initial lepton direction, in the frame in which the proton is at rest, and

$$
\cos \theta_{\gamma}=\frac{\left(s-m_{N}^{2}\right) y+2 m_{N}^{2} x y}{\left[\left(s-m_{N}^{2}\right)^{2} y^{2}+4 m_{N}^{2}\left(s-m_{N}^{2}\right) x y\right]^{1 / 2}} \simeq 1-\frac{2 m_{N}^{2} x^{2}}{Q^{2}}(1-y) .
$$

In the last expression we have neglected terms of $O\left(m_{N}^{4} / Q^{4}\right)$.

Eq. (36) shows that, in this case, $P$ varies with $\phi_{P}^{\ell^{\prime}}$, that is with the orientation of the initial transverse polarization around $\boldsymbol{\ell}$, which is known experimentally, event 
by event. However, one sees also that, with varying $\phi_{P}^{\ell^{\prime}}$, one always has $P_{T} \cos \theta_{\gamma} \leq$ $P \leq P_{T}$ and that, on the average (assuming all values of $\phi_{P}^{\ell^{\prime}}$ to be equally probable):

$$
\left\langle P^{2}\right\rangle=\frac{P_{T}^{2}}{2}\left(1+\cos ^{2} \theta_{\gamma}\right) \text {. }
$$

The factor $P \sin \Phi_{C}$ for a transverse proton polarization, assuming $P_{T}=1$, can then be approximated, using Eqs. (20) and (38), as

$$
P \sin \Phi_{C} \simeq\left[1-\frac{2 m_{N}^{2} x^{2}}{Q^{2}}(1-y)\right] \sin \left(\varphi_{P}+\varphi_{h}\right) .
$$

Notice anyway from Eq. (37) that $\cos \theta_{\gamma}$, for a $Q^{2}$ of a few $(\mathrm{GeV} / c)^{2}$, is very close to 1 so that in practice $P \simeq P_{T}$.

By comparing Eqs. (35) and (39) one sees that for longitudinally polarized protons even the leading twist contribution to $A_{N}$, Eq. (19), is suppressed by powers of $m_{N} / Q$, so that higher twist contributions might be equally important [9, 11]. For transversely polarized protons, instead, the leading twist contribution is dominant, as in this case $P \simeq P_{T}$.

\section{Estimates of the quark analysing power}

We consider now the case of $\pi^{+}$and $\pi^{-}$production with a transversely polarized proton, as measured by SMC collaboration [2], or with a longitudinally polarized proton, as measured by HERMES collaboration [1].

The leading twist expressions for $A_{N}^{\pi^{i}}$, Eq. (24) or its integrated versions, Eq. (29), depend on the transverse spin distribution functions $h_{1 q}$ which are not known: however, one can derive an upper limit for the magnitude of $h_{i}$ or $I_{N_{i}}$ by exploiting the accurate knowledge of the unpolarized distribution functions, $q=f_{q / p}$, of the longitudinally polarized ones, $\Delta q$, and the Soffer bound on $h_{1 q}$ [14]:

$$
\left|h_{1 q}\right| \leq \frac{1}{2}(q+\Delta q) .
$$

This allows, by comparison with the available data on $A_{N}^{\pi}$, to derive a lower bound estimate for the quark analysing power $A_{q}^{\pi}$. We do this, considering separately the SMC and HERMES data, and making some further comments on the latter.

SMC data, transverse proton polarization, Fig. 5

Ref. [2] reports preliminary results on $A_{N}^{h}$ for positive and negative hadrons (mainly pions) produced in the DIS scattering of unpolarized muons off a transversely polarized proton target at:

$$
\langle x\rangle \simeq 0.08 \quad\left\langle Q^{2}\right\rangle \simeq 5(\mathrm{GeV} / c)^{2} \quad\langle z\rangle \simeq 0.45
$$

which implies $\langle y\rangle \simeq 0.33$, as $s=188.5(\mathrm{GeV})^{2}$. They have selected two data sets with $\left\langle p_{T}\right\rangle=0.5 \mathrm{GeV} / c$ and $\left\langle p_{T}\right\rangle=0.8 \mathrm{GeV} / c$; it is interesting to note that $\left|A_{N}^{h}\right|$ 
increases (moderately) with $p_{T}$. They present results for the asymmetry divided by the depolarization factor and for the total amount of events they have:

$$
\begin{aligned}
& \left(A_{N}^{\pi^{+}}\right)_{H}=\frac{1+(1-y)^{2}}{2(1-y)} A_{N}^{\pi^{+}}=-(0.11 \pm 0.06) \sin \left(\varphi_{P}+\varphi_{h}\right) \\
& \left(A_{N}^{\pi^{-}}\right)_{H}=\frac{1+(1-y)^{2}}{2(1-y)} A_{N}^{\pi^{-}}=+(0.02 \pm 0.06) \sin \left(\varphi_{P}+\varphi_{h}\right) .
\end{aligned}
$$

Notice that the choice of axes in Ref. 22 is the same as ours, but they define the Collins angle [see their Eq. (2)] with an opposite sign with respect to our definition, Eqs. (2) and (20).

Evaluating the depolarization factor at $\langle y\rangle \simeq 0.33$, Eqs. (42) and (43) imply:

$$
\begin{aligned}
& A_{N}^{\pi^{+}}=-(0.10 \pm 0.06) \sin \left(\varphi_{P}+\varphi_{h}\right) \\
& A_{N}^{\pi^{-}}=+(0.02 \pm 0.06) \sin \left(\varphi_{P}+\varphi_{h}\right) .
\end{aligned}
$$

On the other hand Eqs. (24)-(26), (39) and (40), evaluated at the average kinematical values of Eq. (41), with the polarized and unpolarized quark distributions of Ref. [15], yield:

$$
\begin{aligned}
& \left|A_{N}^{\pi^{+}}\left(\langle z\rangle, \Phi_{C},\left\langle p_{T}\right\rangle\right)\right| \lesssim 0.41\left|A_{q}^{\pi}\left(\langle z\rangle,\left\langle p_{T}\right\rangle\right) \sin \left(\varphi_{P}+\varphi_{h}\right)\right| \\
& \left|A_{N}^{\pi^{-}}\left(\langle z\rangle, \Phi_{C},\left\langle p_{T}\right\rangle\right)\right| \lesssim 0.11\left|A_{q}^{\pi}\left(\langle z\rangle,\left\langle p_{T}\right\rangle\right) \sin \left(\varphi_{P}+\varphi_{h}\right)\right| .
\end{aligned}
$$

From Eqs. (46) and (44) one has, assuming $h_{1 u}$ to be positive, as all models indicate:

$$
A_{q}^{\pi}\left(\langle z\rangle,\left\langle p_{T}\right\rangle\right) \lesssim-(0.24 \pm 0.15) \quad\langle z\rangle \simeq 0.45, \quad\left\langle p_{T}\right\rangle \simeq 0.65 \mathrm{GeV} / c .
$$

The experimental result (45) is not statistically significant to allow an independent evaluation of a bound on $A_{q}^{\pi}$; however Eqs. (47) and (48) are in agreement with (45).

The estimate (48) is obtained by taking average values of the various kinematical variables, according to the indications of Ref. [1]; we have also redone the above evaluation by explicitely performing the $x$ and $y$ integration,

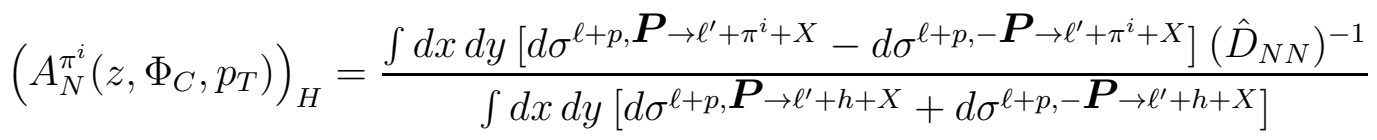

$$
\begin{aligned}
& =\frac{\int d x d y h_{i}(x)\left[1+(1-y)^{2}\right] /\left(x y^{2}\right) P}{\int d x d y f_{i}(x)\left[1+(1-y)^{2}\right] /\left(x y^{2}\right)} A_{q}^{\pi}\left(z, p_{T}\right) \sin \Phi_{C},
\end{aligned}
$$

over the range $x \geq 0.0053 / y=Q_{\min }^{2} /(y s), \quad 0.1 \leq y \leq 0.7, \quad Q^{2} \geq 1(\mathrm{GeV} / c)^{2}$ (results are almost independent of the upper $x$ limit).

From Eqs. (49), (25), (26), (39) and the bound (40), we obtain now:

$$
\begin{aligned}
& \left|\left(A_{N}^{\pi^{+}}\left(\langle z\rangle, \Phi_{C},\left\langle p_{T}\right\rangle\right)\right)_{H}\right| \lesssim 0.42\left|A_{q}^{\pi}\left(\langle z\rangle,\left\langle p_{T}\right\rangle\right) \sin \left(\varphi_{P}+\varphi_{h}\right)\right| \\
& \left|\left(A_{N}^{\pi^{-}}\left(\langle z\rangle, \Phi_{C},\left\langle p_{T}\right\rangle\right)\right)_{H}\right| \lesssim 0.11\left|A_{q}^{\pi}\left(\langle z\rangle,\left\langle p_{T}\right\rangle\right) \sin \left(\varphi_{P}+\varphi_{h}\right)\right|
\end{aligned}
$$


to be compared with Eqs. (42) and (43). This yields,

$$
A_{q}^{\pi}\left(\langle z\rangle,\left\langle p_{T}\right\rangle\right) \lesssim-(0.26 \pm 0.14) \quad\langle z\rangle \simeq 0.45, \quad\left\langle p_{T}\right\rangle \simeq 0.65 \mathrm{GeV} / c
$$

in agreement with (48).

The conclusion induced by SMC data, Eq. (48) or (52), is remarkable and very interesting; the quark analysing power as defined in Eq. (雨) is large (in magnitude) and negative; what is even more remarkable is the fact that the same qualitative conclusion was obtained in Ref. [7], by fitting the data on $p p^{\uparrow} \rightarrow \pi X$, assuming that the observed single spin asymmetries [1] are entirely due to Collins effect. Actually, predictions for $A_{N}^{\pi}$ in DIS, using the evaluation of $\Delta^{N} D_{\pi / q}$ of Ref. [7] were computed in Ref. [16]; however, they were given in different kinematical regions and cannot be compared directly with the data now available.

HERMES data, longitudinal proton polarization, Fig. 4

Ref. [1] reports results on $A_{N}^{\pi}$ for positive and negative pions produced in the DIS scattering of unpolarized positrons off a transversely polarized proton target at $s=52.6(\mathrm{GeV})^{2}$ and in the kinematical ranges:

$$
0.023 \leq x \leq 0.4 \quad 0.1 \leq y \leq 0.85 \quad Q^{2} \geq 1(\mathrm{GeV} / c)^{2} \quad 0.2 \leq z \leq 0.7 .
$$

They give:

$$
\begin{aligned}
& A_{N}^{\pi^{+}} \simeq+[0.022 \pm 0.004(\text { stat. }) \pm 0.004(\text { syst. })] \sin \varphi_{h} \\
& A_{N}^{\pi^{-}} \simeq-[0.001 \pm 0.005(\text { stat. }) \pm 0.004(\text { syst. })] \sin \varphi_{h} .
\end{aligned}
$$

Although they do not take into account the change of direction of $\boldsymbol{P}_{q}$ during the elementary interaction, Eq. (7), their definition of $\sin \Phi_{C}$ agrees with ours, Eq. (34).

There seems to be a sign discrepancy between the SMC and HERMES data; this should be investigated carefully and a consistent definition of the Collins angle used in all experiments.

One should not forget the previous comments about the fact that other higher twist contributions, like those related to the quark transverse spin distribution inside a longitudinally polarized proton [8, 9, 12], are expected to be as important as the leading twist ones, due to Eq. (33): this might indeed change the sign of $A_{N}$ observed by HERMES, with respect to that observed by SMC, as they may originate from different contributions. A confirmation of the sign difference would indeed confirm such situation.

Despite these considerations we proceed now as in the case of transversely polarized protons, and use the upper bound (40), to obtain an upper limit on the value of the leading twist Eq. (29). This in turns results in a lower limit on $A_{q}^{\pi}$. Although this procedure is now much less reliable than in the case of transversely polarized protons - for which leading twist contributions dominate - still it should give us an order of magnitude estimate of the value of $\left|A_{q}^{\pi}\right|$, assuming that leading twist contributions are of the same order as non leading ones. 
From Eqs. (29)-(31), (25)-(26) and (35), together with the bound (40), using the distribution functions of Ref. [15], and integrating over the kinematical range of Eq. (53) one obtains:

$$
\begin{aligned}
& \left|A_{N}^{\pi^{+}}\left(z, \Phi_{C}, p_{T}\right)\right| \lesssim 0.111\left|A_{q}^{\pi}\left(z, p_{T}\right) \sin \varphi_{h}\right| \\
& \left|A_{N}^{\pi^{-}}\left(z, \Phi_{C}, p_{T}\right)\right| \lesssim 0.026\left|A_{q}^{\pi}\left(z, p_{T}\right) \sin \varphi_{h}\right|
\end{aligned}
$$

By comparing Eqs. (56) and (54) one has:

$$
\left|A_{q}^{\pi}\left(z, p_{T}\right)\right| \gtrsim 0.20 \pm 0.04(\text { stat. }) \pm 0.04(\text { syst. }) \quad z \geq 0.2
$$

which, with all uncertainties discussed, indicates again a large value of $\left|A_{q}^{\pi}\right|$. Data on $\pi^{-}$have much too large errors to allow any significant numerical evaluation; however, Eqs. (58) and (57) are consistent with Eq. (55).

\section{Conclusions}

The data [1, 2] on the azimuthal dependence of pions produced in semi-inclusive DIS, with unpolarized leptons and polarized protons, are extremely interesting and, if confirmed, would reveal a new fundamental spin property of the quark fragmentation; this new property might also be responsible for other transverse single spin asymmetries observed in $p p$ inclusive processes [4. A phenomenological approach [7] to these intriguing and subtle experimental measurements seems now indeed possible.

We have analysed SMC and HERMES data within the leading twist QCDfactorization scheme, allowing for intrinsic $\boldsymbol{p}_{T}$ and spin dependence in the fragmentation process of a transversely polarized quark, the so-called Collins effect [3]. This is certainly and largely the dominant contribution in case of transversely polarized protons (SMC), whereas in case on longitudinal polarization (HERMES) higher twist contributions might be equally or even more important.

At leading twist, the measured single spin asymmetries depend on the product of two unknown functions, which contain the quark analysing power $A_{q}^{\pi}$, Eq. (四), and the transverse spin distributions $h_{1 q}$. The first unavoidable conclusion from the data is that such a product has to be large. By exploiting the existence of an upper bound on $\left|h_{1 q}\right|$ we are able to derived a lower limit for the magnitude of $A_{q}^{\pi}$. The SMC data - for which a leading twist analysis is well accurate - indicate a large lower bound on $\left|A_{q}^{\pi}\right|$ and a negative sign for it, in exact agreement with the findings of Ref. [7], where Collins effect was used to describe single transverse spin asymmetries in $p p^{\uparrow} \rightarrow \pi X$ processes.

The HERMES data should be analysed taking into account higher twist contributions, as other authors do [8]-[12]; nevertheless, they also point towards a large value of $\left|A_{q}^{\pi}\right|$. It is interesting to notice that the asymmetries measured by SMC and HERMES, if a consistent definition of the Collins angle is used, appear to differ in sign: the reported data have the same signs, but the definitions of $\sin \Phi_{C}$ used 
by the two collaborations seem to be opposite. If such a difference persists one has to conclude that the origin of the asymmetry measured by HERMES is still dominated by higher twist contributions, and these give opposite (and larger) values with respect to the leading twist contributions.

New data on single transverse spin asymmetries in semi-inclusive DIS and other processes will be available from several experiments in progress or planned; a consistent way of understanding and predicting them in terms of new, fundamental, spin and transverse momentum dependent, fragmentation and distribution functions appears possible.

\section{Acknowledgements}

We would like to thank M. Boglione, A. Bravar and E. Leader for useful discussions. 


\section{References}

[1] HERMES Collaboration (A. Airapetian et al.), DESY-99-149, Oct. 1999, e-Print Archive: hep-ex/9910062; H. Avakian (on behalf of the HERMES collaboration), Nucl. Phys. B79 (Proc. Suppl.) (1999) 523

[2] A. Bravar (on behalf of the SMC collaboration), Nucl. Phys. B79 (Proc. Suppl.) (1999) 520

[3] J.C. Collins, Nucl. Phys. B396 (1993) 161

[4] D.L. Adams et al., Phys. Lett. B264 (1991) 462; Phys. Rev. Lett. 77 (1996) 2626

[5] D. Sivers, Phys. Rev. D41 (1990) 83; D41 (1991) 261

[6] M. Anselmino, M. Boglione and F. Murgia, Phys. Lett. B362 (1995) 164;

M. Anselmino and F. Murgia, Phys. Lett. B442 (1998) 470

[7] M. Anselmino, M. Boglione and F. Murgia, Phys. Rev. D60 (1999) 054027

[8] A.M. Kotzinian, K.A. Oganessyan, H.R. Avakian and E. De Sanctis, e-Print Archive: hep-ph/9908466

[9] D. Boer, e-Print Archive: hep-ph/9912311

[10] A.V. Efremov, K. Goeke, M.V. Polyakov and D. Urbano, e-Print Archive: hepph/0001119

[11] E. De Sanctis, W.-D. Nowak and K.A. Oganessyan, e-Print Archive: hep-ph/0002091; this paper appeared after completion of ours

[12] M. Boglione and P. Mulders, e-Print Archive: hep-ph/0001196

[13] M. Boglione and E. Leader, e-Print Archive: hep-ph/9911207

[14] J. Soffer, Phys. Rev. Lett. 74 (1995) 1292

[15] M. Glück, E. Reya and W. Vogelsang, Z. Phys. C67 (1995) 433; M. Glück, E. Reya M. Stratmann and W. Vogelsang, Phys. Rev. D53 (1996) 4775

[16] M. Anselmino, M. Boglione, J. Hansson and F. Murgia, e-Print Archive: hepph/9906418, to appear in Eur. Phys. J. C 


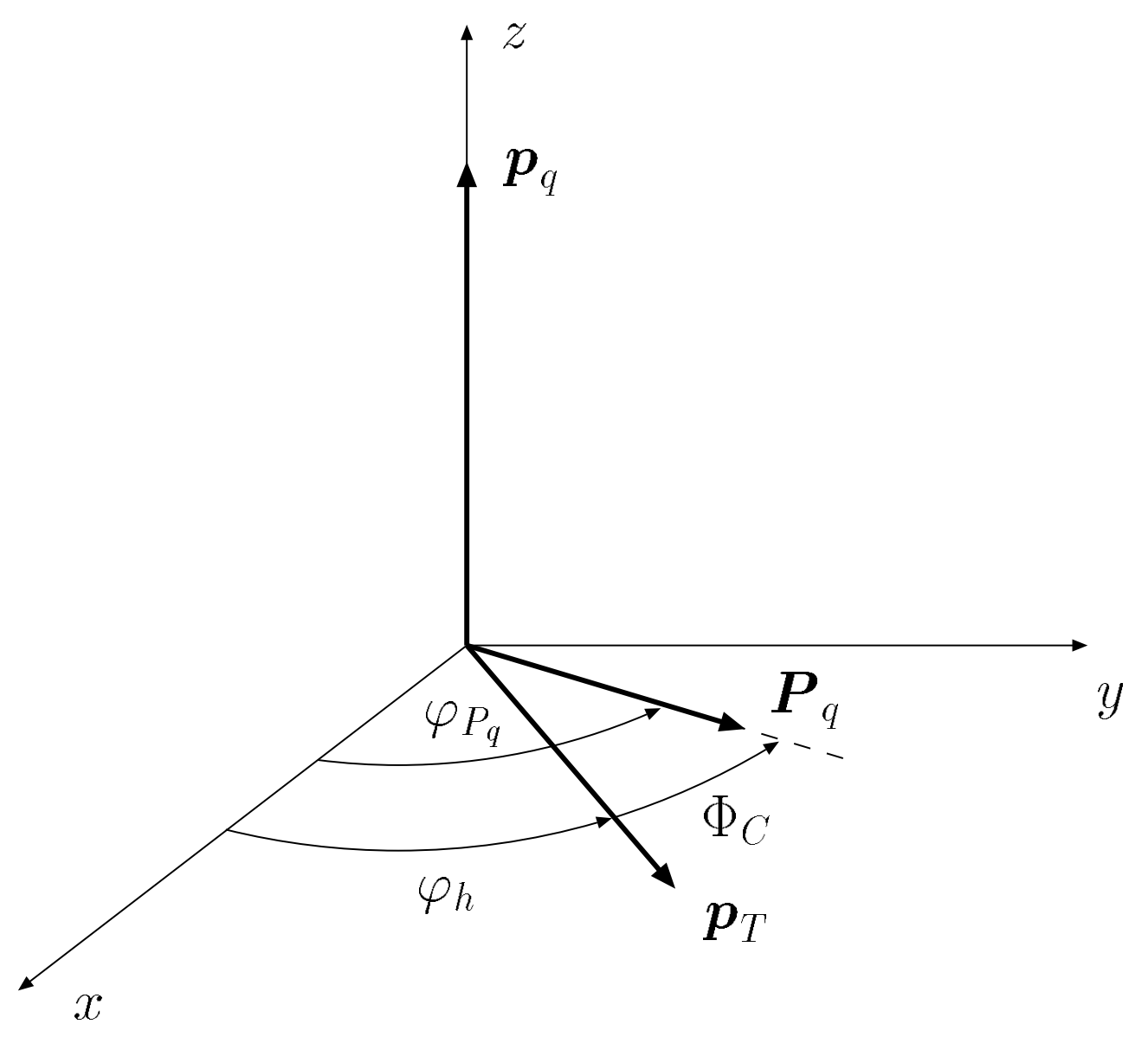

Fig. 1: Definition of the Collins angle for the fragmentation of a quark with momentum $\boldsymbol{p}_{q}$ and transverse spin-polarization vector $\boldsymbol{P}_{q}$ into a hadron with momentum $\boldsymbol{p}_{h}=z \boldsymbol{p}_{q}+\boldsymbol{p}_{T}: \boldsymbol{P}_{q} \cdot\left(\hat{\boldsymbol{p}}_{q} \times \hat{\boldsymbol{p}}_{T}\right)=P_{q} \sin \left(\varphi_{P_{q}}-\varphi_{h}\right) \equiv P_{q} \sin \Phi_{C}$. 


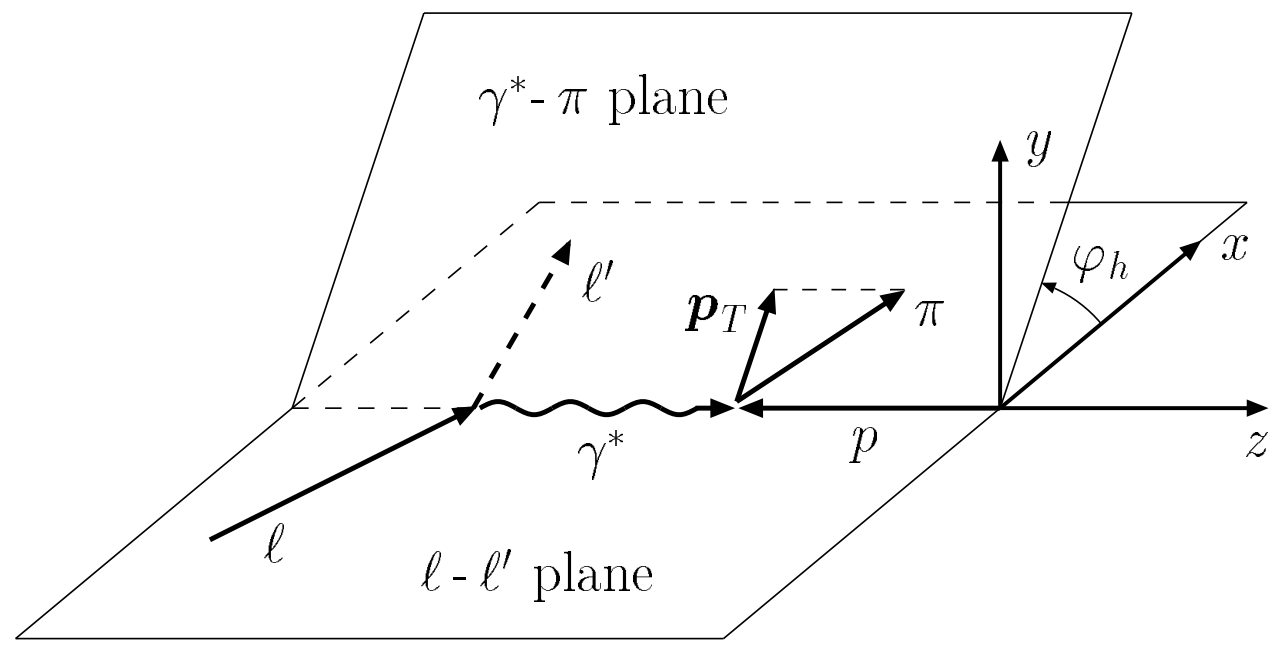

Fig. 2: Definition of our kinematical configuration and choice of the reference frame. 


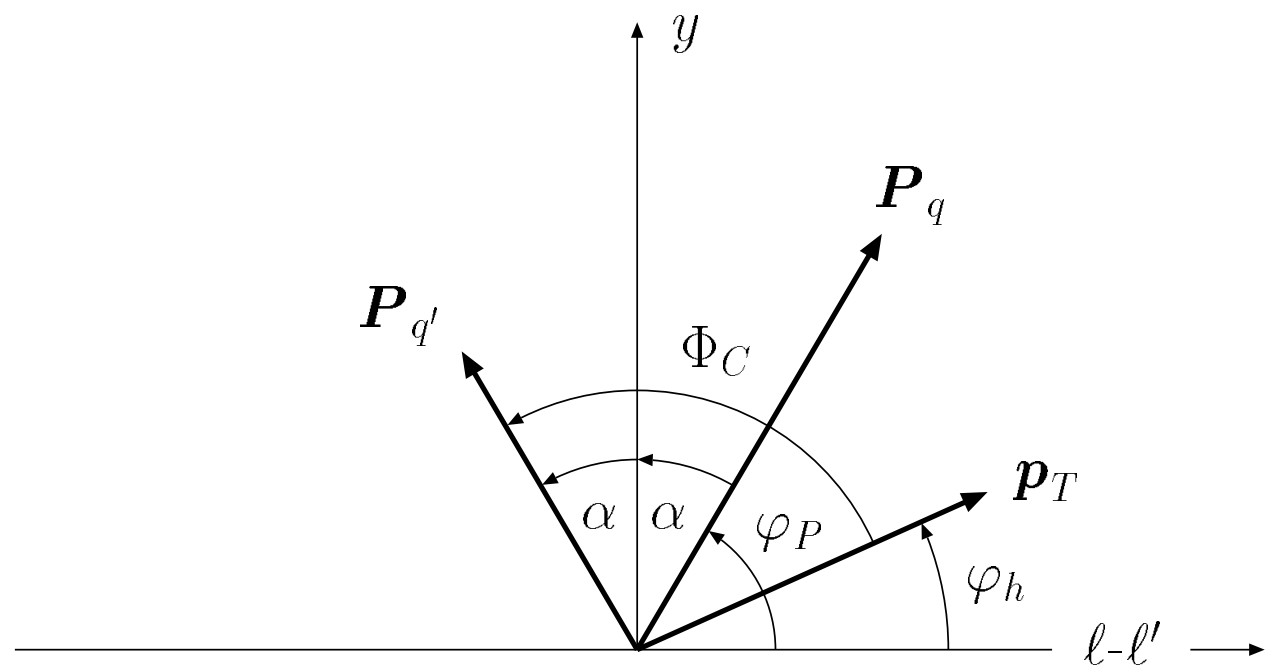

Fig. 3: Transverse polarization vector $\boldsymbol{P}_{q^{\prime}}$ resulting from the $\ell+q, \boldsymbol{P}_{q} \rightarrow$ $\ell^{\prime}+q^{\prime}$ interaction: the final polarization $\boldsymbol{P}_{q^{\prime}}$ is reduced in magnitude by the depolarization factor, $\left|\boldsymbol{P}_{q^{\prime}}\right|=\hat{D}_{N N}\left|\boldsymbol{P}_{q}\right|$ and is symmetric, with respect to the $y$-axis, to $\boldsymbol{P}_{q}$. The $\gamma^{*}$ moves along the positive $z$-axis and the $\ell-\ell^{\prime}$ plane is the $x-z$ plane. Notice that $\Phi_{C}=\varphi_{P_{q^{\prime}}}-\varphi_{h}=\varphi_{P}+2 \alpha-\varphi_{h}=\pi-\varphi_{P}-\varphi_{h}$. 


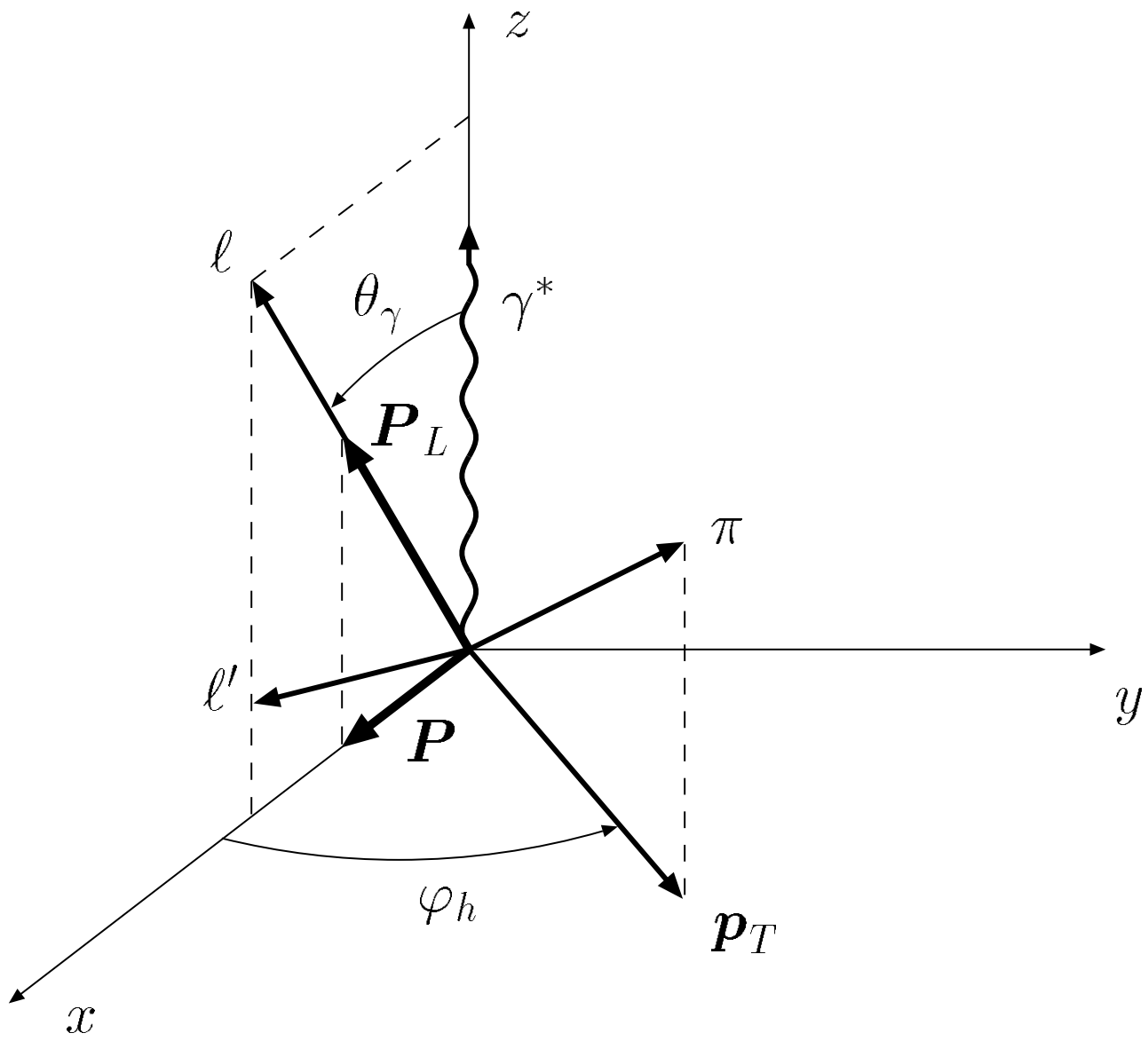

Fig. 4: Kinematical configuration for HERMES experiment, in a frame where the proton is at rest and $\gamma^{*}$ moves along the positive $z$-axis. In this case $P=P_{L} \sin \theta_{\gamma}$ and the Collins angle simplifies to $\Phi_{C}=\pi-\varphi_{P}-\varphi_{h}=\pi-\varphi_{h}$ (the polarization vector of the final fragmenting quark points opposite to $\boldsymbol{P}$ ). 


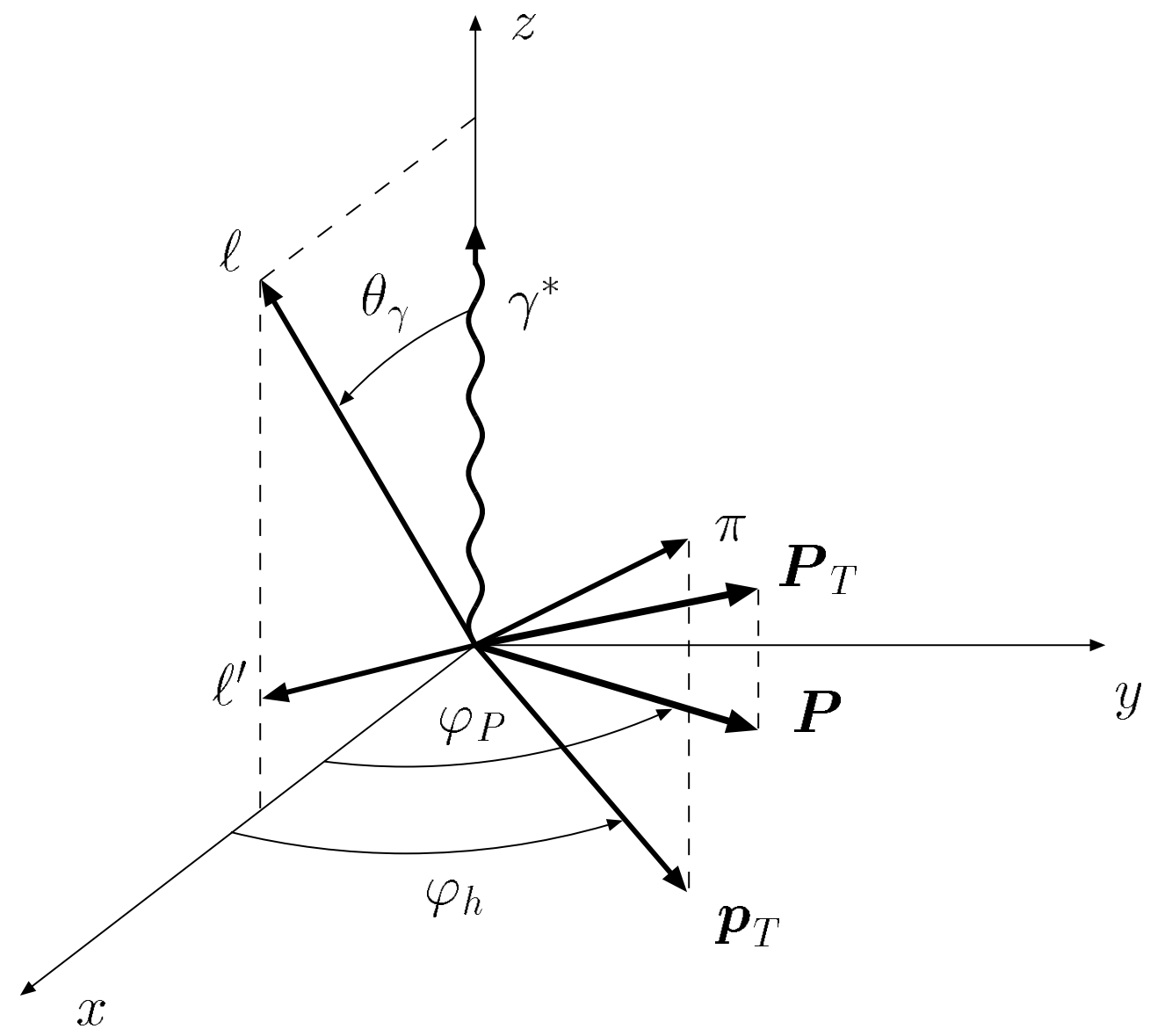

Fig. 5: Kinematical configuration for SMC experiment, in a frame where the proton is at rest and $\gamma^{*}$ moves along the positive $z$-axis. The proton polarization vector $\boldsymbol{P}_{T}$ is now orthogonal to $\boldsymbol{\ell}$; the polarization vector of the final fragmenting quark has direction symmetric to $\boldsymbol{P}$ with respect to the $y$-axis and $\Phi_{C}=\pi-\varphi_{P}-\varphi_{h}$. 